\title{
A study of household contacts of children with leprosy
}

\author{
N P Madarasingha ${ }^{1}$, J K K Senaviratne ${ }^{2}$ \\ (Index words: leprosy, contact tracing)
}

\begin{abstract}
Introduction The new case detection rate of leprosy and new cases among children remain high in Sri Lanka indicating ongoing transmission. Identification of the positive contacts and the source of infection would break this chain of transmission. Contact tracing is known to identify early disease and thus prevent disabilities. However, in the recent past little emphasis has been laid on contact tracing by the health care providers.

Objectives This study looked at the household contacts of children with leprosy to identify the rate of positive contacts within the household.

Methods The study was conducted at the Lady Ridgeway Hospital, Colombo, Sri Lanka, during a period of one year and nine months from January 2007 . The index cases were defined as children of less than 12 years who were presently on anti leprosy treatment or who were newly diagnosed with leprosy. A total of 311 contacts of 100 index cases were examined for evidence of leprosy.

Results The total of positive contacts was 51 per 100 index cases. $33 \%$ of the index cases had a positive contact within the household. $11 \%$ had more than one member affected. $83.2 \%$ of positive contacts were of tuberculoid type. $20.8 \%$ of the contacts were less than 15 years of age. When considering the relationship to the index case, most $(33.3 \%)$ were siblings while $25.0 \%$ were parents and $20.8 \%$ were grandparents. Twenty five persons $(8.0 \%)$ out of 311 household contacts were de novo cases.
\end{abstract}

Conclusions This study highlights the value of contact screening of leprosy patients.

Ceylon Medical Journal 2011; 56: 112-114

\section{Introduction}

Leprosy is a chronic disease leading to disability and loss of functional independence. Early detection and treatment is important in preventing complications. Sri Lanka has reached the WHO elimination targets in 1995. However, the number of new cases detected remains around 2000 per year and the rate of detection of new cases among children is high $(10.2 \%$ in 2007 and $10.3 \%$ in 2008 ) indicating ongoing transmission $[1,2]$.
Leprosy is a disease with a long incubation period. This makes identification of the possible source of infection difficult. Transmission of leprosy is thought to be primarily person-to-person by air borne spread. The risk of developing leprosy is 5-10 times higher if one member of the family has developed the disease previously and higher if the primary case has lepromatous leprosy and lower if tuberculoid leprosy [3].

Identification of the possible source of infection and a better understanding of the mode of transmission will help the future eradication strategies. The main objective of this study was to identify the positive contacts within the households.

\section{Methods}

The study was conducted at the Lady Ridgeway Hospital, Colombo, Sri Lanka, during a period of one year and nine months from January 2007. The index cases were defined as children of less than 12 years who were presently on anti leprosy treatment or who were newly diagnosed with leprosy. The diagnosis of leprosy in the index cases was made mainly on clinical grounds. In some instances the diagnosis was confirmed by characteristic histological changes or positive skin smear. The household contacts were defined as persons who live together with the index case or frequent visitors to the household. All contacts who volunteered to participate were included in the study. Verbal consent was obtained from the participants following explanation of the nature and the spread of the disease and the objectives of the study. For participants below 12 years consent was obtained from the parent or the legal guardian. Approval for the study was obtained from the ethical review committee of the Lady Ridgeway Hospital.

An interviewer administered questionnaire was used to record the details of the index case and the contact. For children who were already on treatment additional information was taken from the clinic records. All contacts were examined by the authors for clinical evidence of leprosy. Skin biopsies were performed in all clinically suspicious lesions and skin smears were done in some cases.

\footnotetext{
${ }^{1}$ Dermatology Unit, National Hospital of Sri Lanka, ${ }^{2}$ Dermatology Unit, The Lady Ridgeway Hospital for Children, Colombo, Sri Lanka.
}

Correspondence: NPM, e-mail: <nayanimadara@yahoo.com>. Received 1 October 2010 and revised version accepted 24 March 2011. Competing interests: none declared. 


\section{Results}

A total of 100 index cases were included of which $62 \%$ were males. $96 \%$ of the index cases had polar tuberculoid (TT) leprosy (the majority having a single patch) or borderline tuberculoid leprosy (BT). Only 4\% had borderline lepromatouos leprosy $(\mathrm{BL})$. There were no polar lepromatous (LL) patients.

The total number of contacts identified for 100 index cases was 344. Out of them $311(90.40 \%)$ volunteers were examined for clinical evidence of leprosy.

A total number of 51 positive contacts were detected among the household contacts of 100 index cases. 33\% of the index cases had a positive contact. $11 \%$ had more than one positive contact (range 2-4). Forty four (83.2\%) of the positive contacts were of TT/BT leprosy. 18 (35.3\%) of the positive contacts were less than 15 years of age. When considering the relationship to the index case, most (33.3\%) were siblings while $25.0 \%$ were parents and $20.8 \%$ were grandparents. Out of the 51 positive contacts $15.7 \%$ were currently on treatment, $31.3 \%$ had completed therapy, $3.9 \%$ were defaulters and $49.0 \%$ were detected as new cases. It is important to note that twenty five persons $(8.0 \%)$ out of a total of 311 household contacts examined were detected as de novo cases.

\section{Discussion}

Although Sri Lanka has achieved the leprosy elimination target of the WHO in 1995, the new case detection rate has been static over the past decade and the childhood leprosy rate remains high $[1,2]$. Leprosy is found in clusters and screening of contacts will help to determine the source of infection and to break the chain of transmission.

A previous study in Sri Lanka showed that 20\% $(148 / 726)$ of the leprosy patients had more than one leprosy patient in the family with a positive contact rate of $4.8 \%$ among household contacts [4]. Our study showed relatively higher values with $33 \%$ of the index cases having at least one positive contact with $16.4 \%$ of the household contacts having leprosy.

However, similar results have been observed in some other countries. A study done in India showed that a history of contact was present in $38.8 \%$ with $95 \%$ of them being within the family [5]. A Brazilian study revealed that $44.7 \%$ reported a known leprosy contact and $14 \%$ were within the household [6]. The household contact rate is much higher in countries where leprosy is not endemic [6]. A study of children with leprosy done in China showed that $70.0 \%$ of the affected cases were infected by intrafamilial contact [7]. All these findings emphasise the importance of contact tracing in the control of leprosy.

Although the 7th WHO Expert Committee in June 1997 had recommended that the household contacts of the patient should be examined for evidence of leprosy, implementing this strategy has been limited due to ethical considerations, fear of exacerbating stigma and difficulties in implementation [8].

In our study, out of 51 positive contacts $25(49.0 \%)$ were detected as new patients. The chances of detection were high as all contacts were carefully examined by the authors. Most studies in the literature have concentrated on the index case and looked for the transmission rate to contacts in the future [6]. We regarded the index case as the secondary case and tried to locate other positive contacts within the household. The source of infection could not be identified exactly from this study. However, the new case detection rate was $8.03 \%$ in the contact population when compared to the national figure of approximately 10 per 100,000 of population [9]. This highlights that the household contacts are at a higher risk of developing the disease and the value of screening contacts of all leprosy patients. These findings further strengthens the WHO global strategy agreed for 20112015 , to examine all household contacts of newly detected patients as a positive step forward to detect cases early.

The study being a cross sectional one, the dynamic nature of the disease was not assessed. Some of the new cases we have detected may turn out to be indeterminate leprosy. At the same time the possibility of a percentage of the household contacts that were negative for leprosy developing the disease later was not assessed. The sample bias of recruiting the volunteers who may be symptomatic was minimised by the high participation rate $(90.04 \%)$ of the contact sample.

An interesting feature of the results was that $86.3 \%$ of positive contacts and $96 \%$ of the index cases were TT/ BT type of leprosy. A study done in Northern Malawi revealed that the individuals living in household or dwelling in contact with paucibacillary cases were at approximately two-fold increased risk compared with individuals not living in such households or dwellings [3]. But this would not explain the rate of TT/BT contacts found in our study. One possibility for a higher risk is exposure to a common source of infection outside the household. As the source of infection was not revealed from this study this should extend to the neighbourhood and other social contacts as well. Genetic predisposition to development of the disease is another explanation [10]. The findings of multiple positive contacts in $11 \%$ of the index cases and $20.8 \%$ of positive contacts being below 15 years favours genetic susceptibility to contract disease. These findings also raise the question of other modes of transmission of leprosy bacilli which are yet to be proved.

\section{Conclusions}

This study emphasises the fact that household contacts of leprosy patients are at a greater risk of developing the disease and that the possibility of new case detection is significantly higher in the contact population than the normal population. The health care 
providers and policy planners in leprosy control programmes should pay more attention to contact tracing of all leprosy patients, although it is a disease which has reached the $\mathrm{WHO}$ elimination targets.

\section{Acknowledgements}

The authors acknowledge the valuable support given by the staff of the Dermatology Unit, Lady Ridgeway Hospital for Children and by Dr. Indira Kahawita, Consultant Dermatologist, General Hospital, Polonnaruwa.

\section{References}

1. http://www.who.int/wer/2007/wer8251_52/en/index.html accessed on $08 / 09 / 2010$.

2. http://www.who.int/wer/2009/wer8433/en/index.html accessed on $08 / 09 / 2010$.

3. Fine PE, Sterne JA, Ponnighaus JM, et al. Household and dwelling contact as risk factors for leprosy in northern Malawi. American Journal of Epidemiology 1997; 146: 91-102.
4. Dissanayake S. Relative lack of clinical disease among household contacts of tuberculosis patients compared to leprosy households. Transactions of the Royal Society of Tropical Medicine and Hygiene 2004; 98: 156-64.

5. Jain S, Reddy RG, Osmani SN, et al. Childhood leprosy in an urban clinic, Hyderabad, India: clinical presentation and the role of household contacts. Leprosy Review 2002; 73: 248-53.

6. Deps PD, Guedes BVS, Jander BF, et al. Characteristics of known leprosy contacts in a high endemic area in Brazil. Leprosy Review 2006; 77: 34-40.

7. Chen X, Li W, Jiang C, Ye G. Leprosy in children: a retrospective study in China, 1986-1997. Journal of Tropical Pediatrics 2000; 46: 207-11.

8. http://www.searo.who.int/EN/Section980/Section2572/ Section2578_14961.htm accessed on 07/09/2010.

9. http://www.searo.who.int/LinkFiles/Reports_LEP-166.pdf accessed on 08/09/2010.

10. Schuur E, Alcais A, de Leseleucl, Abel L. Genetic predisposition to leprosy: a major gene reveals novel pathways of immunity to Mycobacterium leprae. Seminars in Immunology 2006; 18: 404-10. 